\title{
The Value of Sampling Inspection in a Single-Period Remanufacturing System with Stochastic Returns Yield
}

\author{
Christos Zikopoulos, Sofia Panagiotidou, and George Nenes \\ Department of Mechanical Engineering, Aristotle University of Thessaloniki, \\ 54124 Thessaloniki, Greece, \\ Tel.: +30 231 0995914; Fax: +30 2310996018 \\ cziko@auth.gr, span@auth.gr, gnenes@auth.gr
}

\begin{abstract}
We examine a reverse supply chain consisting of a collection site, where consumers return used products, and a remanufacturing facility. Some of the returned products are transported to the remanufacturing facility in order to be remanufactured and used to satisfy the stochastic demand for remanufactured products. The quality of returns is characterized by uncertainty, and therefore, before the procurement quantity determination, the remanufacturer has the alternative to inspect a sample drawn from the collected quantity in order to evaluate more accurately returns' quality. Using general assumptions for returns quality and remanufactured products demand distributions, we formulate the expected profit function for both sampling and no-sampling cases and we examine numerically the economic effectiveness of sampling. A key characteristic of the current paper is that returns' yield is expressed as the probability of a unit to be remanufacturable.
\end{abstract}

Keywords: reverse supply chain, random yield, sampling inspection, binomial yield, value of information.

\section{Introduction}

One of the most important issues in Reverse Supply Chain Management is the quality of returned used units. Returns quality is associated with the ability of a unit to successfully undergo a recovery process, such as remanufacturing, refurbishing, repair, etc. Firms engaged in value-recovery activities employ a number of different practices in order to obtain information on returns quality. There are two main dimensions regarding this information: accuracy and timing. Usually, the acquisition of timely returns quality information requires the shift of inspection operations at the collection sites. Some remanufacturing firms introduce certain nominal metrics based on specific product characteristics and assign to the supplier the task of inspecting and grading the returned units, e.g. ReCellular [1]. Consequently, the supplier provides information regarding returns quality to the remanufacturer. Another way to obtain timely information is by incorporating electronic devices in the products that record basic usage data, e.g. Bosch [2], HP [3], which provide some information usually indirectly related to the quality of each unit upon its return. Both these practices permit an initial classification of returns according to their quality, before the investment 
of significant resources by the remanufacturer. The disadvantage of these methods is that the accuracy of the information is generally limited. On the other hand, when the accuracy of the returns quality assessment is important, remanufacturing firms prefer to transfer the collected quantity to the remanufacturing site, disassemble all available units and inspect them, e.g. NEC-CI [4], Mercedes-Benz [5]. The obvious disadvantage of this practice is that it can result in a considerable waste of time and effort because of the delayed identification of inferior-quality lots.

In the current paper we propose a different practice; that is to examine a sample taken from the collected quantity and base the procurement decision on the inspection outcome. To the best of our knowledge, sampling inspections in a reverse supply chain context has been initially proposed in [6]. In the current paper we extend the work of [6] for the case of stochastic demand for remanufactured products. The advantage of sampling inspection is that quality assessment can be carried out using a fairly accurate method while the total inspection cost is kept bearable. Of course, due to sampling there are inherent statistical errors.

The uncertainty in returns quality is the main issue in a number of papers as for example in [7], [8], [9], [10] and [11]. The main objective in the aforementioned contributions is either the determination of procurement and remanufacturing decisions or the evaluation of the value of advanced information on returns quality. Other relevant contributions include [12] and [13] in which apart from the procurement and remanufacturing decisions, the impact of grading errors is explored, as well.

The scope of the current paper is to study the advisability of establishing sampling inspection in reverse supply chains. The yield (i.e. the probability that a unit can be remanufactured successfully) of returns is considered stochastic and it is formulated as a continuous random variable. Before determining the procurement quantity, a sample from the collected quantity is inspected. Based on the outcome of this inspection, the prior belief about returns' quality is updated and the optimal procurement quantity is defined. Under general assumptions, we formulate the expected profit functions corresponding to the cases that procurement quantity is decided with or without conducting previously a sampling inspection. The optimal sampling, procurement and remanufacturing decisions are evaluated numerically.

There are two characteristics of the current paper that have not been studied extensively in the reverse supply chain literature:

a) For each returned unit in the procurement lot, it is assumed that there is a specific probability to belong to a certain quality category. Therefore, the number of remanufacturable units in the quantity received is defined as a Binomial random variable. Although this type of random yield model can be found in conventional supply chain literature, e.g. [14], it is rare in the reverse supply chain context.

b) The advisability of conducting sampling inspection is explored, taking into account that the decision of the procurement quantity is based on the inspection outcome. The issue of simultaneous determination of procurement quantity and sampling scheme has already been examined in the context of forward supply chains, e.g. [15] and [16]. Contrary to existing contributions, we examine this issue in the reverse supply chain context. In addition, we treat the sample size as a decision variable and we allow the procurement quantity to vary with respect to the outcome of sampling inspection. 
The remainder of the paper is organized as follows. In the next section we describe in detail the problem setting and we define the basic assumptions. In Section 3 we present the formulation of the expected profit function for the cases without and with sampling inspection. Section 4 presents a numerical study and discusses the findings regarding the impact of the problem parameters on the optimal policy. Finally, Section 5 summarizes and concludes the paper.

\section{Problem Setting and Assumptions}

The reverse supply chain examined consists of a single collection site (CS) and a remanufacturing facility $(\mathrm{R})$. At the collection site, end-users return used products. Each returned unit can be in one of two possible quality states, remanufacturable or non-remanufacturable. Although the actual condition of each unit is unknown, there is a rough knowledge about the quality of the lot collected at the CS. Specifically, we assume that all returned units in the lot have a specific but unknown probability, q, to be remanufacturable. This probability is considered a random variable, which follows a known distribution with density and probability functions $g(q)$ and $G(q)$, respectively.

The remanufacturer, in order to decide the exact amount of returned units to procure, Q, has two alternatives: either to rely on the initial knowledge of returns yield distribution or to inspect a sample of size $\mathrm{n}$ drawn from the quantity collected, at a cost of $c_{n}$ per unit, evaluate the yield in the sample and consequently update the distribution of $\mathrm{q}$ based on the ratio of number remanufacturables in the sample to the sample size $\left(Q_{n} / n\right)$. In either case, when $R$ procures some quantity of returned units, which $\operatorname{cost} c_{a}$ per unit, upon reception it implements a thorough inspection procedure to the total quantity received (e.g. disassemble every unit and check each of its components) in order to identify all remanufacturable units in the procurement quantity. The respective cost at this stage is set equal to $\mathrm{c}_{\mathrm{da}}$.

After disassembly, the exact number of available remanufacturable units, $Q_{a}$, is revealed, and the remanufacturer reaches at a second decision stage which is related to the number of remanufacturable units that will undergo the remanufacturing procedure, $\mathrm{Q}_{\mathrm{r}}$, taking into account the relative cost and revenues as well as the demand characteristics. All units that after disassembly were classified as non-remanufacturables as well as the excess remanufacturables are disposed of at a cost, $c_{d}$ and $c_{d r}$, respectively. The remanufacturing process costs $c_{\mathrm{r}}$ per unit. It is assumed that all remanufactured units can be considered suitable to satisfy demand for remanufactured products.

Demand for remanufactured products is considered a random variable, $\mathrm{x}$, with mean equal to $\mathrm{E}(\mathrm{x})$ and with $\mathrm{f}(\mathrm{x})$ and $\mathrm{F}(\mathrm{x})$ used to denote the density and probability functions, respectively. The sales revenue equals $\mathrm{v}$ per remanufactured unit sold. We assume that $\mathrm{v}>\mathrm{c}_{\mathrm{r}}$, in order to assure that it is worth considering remanufacturing of returns as a profitable option. If demand exceeds the number of available remanufactured units, a cost equal to $\mathrm{c}_{\mathrm{s}}$ per unit short is incurred. Unsold remanufactured units also incur cost, denoted by $c_{u}$ per unit. We assume that $c_{r}+c_{u}>c_{d r}$, since otherwise it would be profitable just to remanufacture returns without the intention to sell them. Table 1 summarizes the notation used throughout the paper. 
Table 1. Notation

\begin{tabular}{|c|c|}
\hline $\begin{array}{ll}\mathrm{v} & \text { Sales revenue per unit }\end{array}$ & Sample size \\
\hline $\mathrm{c}_{\mathrm{a}}$ Acquisition cost per unit & Q Procurement quantity \\
\hline$c_{d a}$ Disassembly cost per unit & $\mathrm{Q}_{\mathrm{r}}$ Number of units to remanufacture \\
\hline$c_{d}$ Disposal cost per non-remanufacturable unit & $\mathrm{Q}_{\mathrm{n}}$ Remanufacturable units in the sample \\
\hline$c_{\mathrm{dr}}$ Disposal cost per remanufacturable unit & $\mathrm{Q}_{\mathrm{a}}$ Available remanufacturables in $\mathrm{Q}$ \\
\hline$c_{\mathrm{r}} \quad$ Remanufacturing cost per unit & q Probability of a unit to be remanufacturable \\
\hline$c_{s}$ Shortage cost per unit short & $\mathrm{x} \quad$ Demand for remanufactured units \\
\hline$c_{u}$ Cost per unsold remanufactured unit & $g(q)$ Probability density function of $q$ \\
\hline$c_{n}$ Inspection cost per unit in the sample & $f(x)$ Probability density function of $x$ \\
\hline
\end{tabular}

\section{Expected Profit Function Formulation}

Regardless of the establishment of sampling inspection, after disassembling the procurement quantity the only remaining factor of uncertainty is demand. In that stage, having resolved quality uncertainty the remanufacturer can determine the optimal quantity that should be remanufactured. The expected profit function at this stage can be written as:

$$
T P\left(Q_{r}\right)=\left(v+c_{s}+c_{u}\right) \int_{0}^{Q_{r}}\left(x-Q_{r}\right) f(x) d x+\left(v+c_{s}+c_{d r}-c_{r}\right) Q_{r}-c_{d r} Q_{a}-c_{s} E(x)
$$

It is easy to show that (1) is maximized for $\mathrm{Q}_{\mathrm{r}}^{*}$, which satisfies:

$$
\mathrm{F}\left(\mathrm{Q}_{\mathrm{r}}^{*}\right)=\left(\mathrm{v}+\mathrm{c}_{\mathrm{s}}+\mathrm{c}_{\mathrm{dr}}-\mathrm{c}_{\mathrm{r}}\right) /\left(\mathrm{v}+\mathrm{c}_{\mathrm{s}}+\mathrm{c}_{\mathrm{u}}\right) \text {. }
$$

Since the quality of returns is uncertain, it is not assured that at the second decision stage there will always be adequate remanufacturable units for processing. Therefore, the optimal policy at this stage is to remanufacture-up-to $Q_{r}^{*}$ units and dispose of the remaining, if there are adequate remanufacturable units. Otherwise, i.e. if $Q_{a} \leq Q_{r}^{*}$, all available units should be remanufactured.

When there is not the alternative of sampling inspection, the lot-sizing decision is based on the prior knowledge for the returns quality. The formulation of the expected profit function is carried out separately depending on the relationship between the values of the procurement quantity, $Q$, and the optimal remanufacturing quantity, $Q_{r}^{*}$. For simplification of exposition we introduce the function B, defined as follows:

$$
B_{a}^{b}(y)=\sum_{Q_{a}=a}^{b} y\left(\begin{array}{c}
Q \\
Q_{a}
\end{array}\right) q^{Q_{a}}(1-q)^{Q-Q_{a}}
$$

Taking into account all relevant costs and revenues and after some algebraic manipulation and using (2), the expected total profit is written as follows:

$$
\begin{gathered}
\mathrm{TP}[\mathrm{Q} \lg (\mathrm{q})]=\left(\mathrm{v}+\mathrm{c}_{\mathrm{s}}+\mathrm{c}_{\mathrm{u}}\right) \int_{0}^{1} \mathrm{~B}_{0}^{\mathrm{Q}}\left(\int_{0}^{\mathrm{Q}_{\mathrm{a}}}\left(\mathrm{x}-\mathrm{Q}_{\mathrm{a}}\right) \mathrm{f}(\mathrm{x}) \mathrm{dx}\right) \mathrm{g}(\mathrm{q}) \mathrm{dq}+\left(\mathrm{v}+\mathrm{c}_{\mathrm{s}}+\mathrm{c}_{\mathrm{d}}-\mathrm{c}_{\mathrm{r}}\right) \mathrm{QE}(\mathrm{q}) \\
-\left(\mathrm{c}_{\mathrm{d}}+\mathrm{c}_{\mathrm{a}}+\mathrm{c}_{\mathrm{da}}\right) \mathrm{Q}-\mathrm{c}_{\mathrm{s}} \mathrm{E}(\mathrm{x}), \quad \text { for } \mathrm{Q} \leq \mathrm{Q}_{\mathrm{r}}^{*}, \text { and }
\end{gathered}
$$




$$
\begin{aligned}
\mathrm{TP}[\mathrm{Q} \mid \mathrm{g}(\mathrm{q})] & =\left(\mathrm{v}+\mathrm{c}_{\mathrm{s}}+\mathrm{c}_{\mathrm{u}}\right) \int_{0}^{1} \mathrm{~B}_{0}^{\mathrm{Q}_{\mathrm{r}}^{*}}\left(\int_{\mathrm{Q}_{\mathrm{r}}^{*}}^{\mathrm{Q}_{\mathrm{a}}}\left(\mathrm{x}-\mathrm{Q}_{\mathrm{a}}\right) f(\mathrm{x}) \mathrm{dx}\right) \mathrm{g}(\mathrm{q}) \mathrm{dq}+\left(\mathrm{v}+\mathrm{c}_{\mathrm{s}}+\mathrm{c}_{\mathrm{u}}\right) \int_{0}^{\mathrm{Q}_{\mathrm{r}}^{*}} \mathrm{xf}(\mathrm{x}) \mathrm{dx} \\
& +\left(\mathrm{c}_{\mathrm{d}}-\mathrm{c}_{\mathrm{dr}}\right) \mathrm{QE}(\mathrm{q})-\left(\mathrm{c}_{\mathrm{d}}+\mathrm{c}_{\mathrm{a}}+\mathrm{c}_{\mathrm{da}}\right) \mathrm{Q}-\mathrm{c}_{\mathrm{s}} \mathrm{E}(\mathrm{x}), \text { for } \mathrm{Q}>\mathrm{Q}_{\mathrm{r}}^{*} .
\end{aligned}
$$

Given the distribution of the yield, the optimal procurement quantity, $\mathrm{Q}^{*}$ can be evaluated using (3) and (4).

When sampling inspection is in effect, the sampling outcome is used to update the estimation regarding the quality of the collected quantity using Bayes theorem. The lot-sizing decisions are based on the posterior distribution of the returns quality. The form of the expected profit function given the sampling outcome is identical to the case without sampling inspection, substituting for $g(q)$ the posterior distribution that is derived based on the sampling inspection outcome and subtracting the cost of the sample inspection. Thus, given the values of $n$ and $Q_{n}$, and the posterior distribution $g_{n, Q_{n}}(q),(3)$ and (4) apply for the expected profit function,

$$
T P\left[Q \mid n, Q_{n}\right]=T P\left[Q \mid g_{n, Q_{n}}(q)\right]-c_{n} n \text {. }
$$

The expected profit for any possible outcome of the sampling inspection can be computed as the expected value of the profit weighted over all possible outcomes of the inspection, which are based on the prior distribution of the yield,

$$
\mathrm{E}_{\mathrm{Q}_{\mathrm{n}}}[\mathrm{TP}(\mathrm{Q})]=\int_{0}^{1} \sum_{\mathrm{Q}_{\mathrm{n}}=0}^{\mathrm{n}}\left\{\mathrm{TP}\left[\mathrm{Q}^{*} \mid \mathrm{g}_{\mathrm{n}, \mathrm{Q}_{\mathrm{n}}}\left(\mathrm{q}_{\mathrm{n}, \mathrm{Q}_{\mathrm{n}}}\right)\right] \mathrm{b}\left(\mathrm{Q}_{\mathrm{n}} ; \mathrm{n}, \mathrm{q}\right)\right\} \mathrm{g}(\mathrm{q}) \mathrm{dq}-\mathrm{c}_{\mathrm{n}} \mathrm{n},
$$

where $\mathrm{b}(\mathrm{x} ; \mathrm{y}, \mathrm{z})$ stands for the probability that a random variable that follows the Binomial distribution with parameter $\mathrm{z}$ takes the value $\mathrm{x}$ after $\mathrm{y}$ Bernoulli trials.

\section{Numerical Illustration and Discussion}

In this section we present a numerical experiment which investigates the economic effectiveness of sampling inspection as well as its interaction with the mean and variance of the yield and the costs of remanufacturing, disassembly and sampling inspection. The random yield is modeled using 6 different distributions of the Beta family. The Beta parameter values were selected so as to examine 3 levels of yield mean, i.e., low $(\mathrm{E}(\mathrm{q})=1 / 3)$, medium $(\mathrm{E}(\mathrm{q})=1 / 2)$ and high $(\mathrm{E}(\mathrm{q})=2 / 3)$, and two levels of yield variance, low $(\mathrm{V}(\mathrm{q})=0.03)$ and high $(\mathrm{V}(\mathrm{q}) \simeq 0.07)$. Remanufacturing and disassembly costs are examined in two levels. Specifically, $c_{\mathrm{r}}$ is set equal to 10 or 20 , while $\mathrm{c}_{\mathrm{da}}$ $=0.25 \mathrm{c}_{\mathrm{r}}$ or $\mathrm{c}_{\mathrm{da}}=\mathrm{c}_{\mathrm{r}}$. The demand is modeled using normal distribution with mean and standard deviation equal to $\mu=100$ and $\sigma=10$, respectively. The sales revenue, $v$, is set in all examples equal to 100 , while the shortage cost, $\mathrm{c}_{\mathrm{s}}$, is assumed negligible. Disposal costs $\left(c_{d}, c_{d r}\right)$ as well as cost of unsold remanufacturable units, $c_{u}$, are set equal to 1 . Finally, acquisition cost, $c_{a}$, is set equal to 15 . Table 2 summarizes the 24 parameter sets used in the numerical investigation. 
Table 2. Parameter values and optimization results

\begin{tabular}{|c|c|c|c|c|c|c|c|c|c|c|}
\hline \multirow[b]{2}{*}{ \# } & \multicolumn{4}{|c|}{ Parameter values } & \multicolumn{2}{|c|}{ No sampling } & \multicolumn{2}{|c|}{$\begin{array}{c}\text { Sampling } \\
\left(\text { low } c_{n}\right)\end{array}$} & \multicolumn{2}{|c|}{$\begin{array}{c}\text { Sampling } \\
\left(\text { high } c_{n}\right)\end{array}$} \\
\hline & $E(q)$ & $\mathrm{V}(\mathrm{q})$ & $\mathrm{c}_{\mathrm{r}}$ & $\mathrm{c}_{\mathrm{da}}$ & $\mathrm{Q}^{*}$ & $\mathrm{TP}$ & $\mathrm{n}^{*}$ & $\%$ & $\mathrm{n}^{*}$ & $\%$ \\
\hline 1 & low & low & 10 & 2.5 & 205 & 1918.7 & 42 & 16.6 & 26 & 13.1 \\
\hline 2 & low & low & 10 & 10 & 153 & 579.8 & 17 & 44.8 & 10 & 37.8 \\
\hline 3 & low & low & 20 & 5 & 167 & 850.2 & 27 & 31.7 & 15 & 26.1 \\
\hline 4 & low & low & 20 & 20 & 0 & 0.0 & 4 & 100.0 & 0 & 0.0 \\
\hline 5 & low & high & 10 & 2.5 & 170 & 1595.2 & 44 & 30.2 & 29 & 27.4 \\
\hline 6 & low & high & 10 & 10 & 127 & 488.8 & 16 & 61.0 & 9 & 57.2 \\
\hline 7 & low & high & 20 & 5 & 138 & 711.0 & 26 & 48.3 & 14 & 44.7 \\
\hline 8 & low & high & 20 & 20 & 0 & 0.0 & 5 & 100.0 & 3 & 100.0 \\
\hline 9 & medium & low & 10 & 2.5 & 189 & 4022.0 & 40 & 4.2 & 22 & 2.5 \\
\hline 10 & medium & low & 10 & 10 & 165 & 2696.3 & 0 & 0.0 & 0 & 0.0 \\
\hline 11 & medium & low & 20 & 5 & 170 & 2723.3 & 18 & 1.6 & 0 & 0.0 \\
\hline 12 & medium & low & 20 & 20 & 125 & 513.3 & 7 & 18.7 & 2 & 8.9 \\
\hline 13 & medium & high & 10 & 2.5 & 154 & 3202.2 & 44 & 13.8 & 25 & 11.9 \\
\hline 14 & medium & high & 10 & 10 & 130 & 2144.9 & 14 & 16.6 & 10 & 12.8 \\
\hline 15 & medium & high & 20 & 5 & 134 & 2163.0 & 23 & 16.0 & 15 & 13.0 \\
\hline 16 & medium & high & 20 & 20 & 101 & 423.3 & 6 & 59.5 & 4 & 55.3 \\
\hline 17 & high & low & 10 & 2.5 & 154 & 5173.1 & 36 & 4.0 & 19 & 2.9 \\
\hline 18 & high & low & 10 & 10 & 140 & 4073.6 & 7 & 0.9 & 0 & 0.0 \\
\hline 19 & high & low & 20 & 5 & 142 & 3874.3 & 16 & 2.3 & 7 & 1.0 \\
\hline 20 & high & low & 20 & 20 & 119 & 1928.7 & 0 & 0.0 & 0 & 0.0 \\
\hline 21 & high & high & 10 & 2.5 & 147 & 4838.5 & 34 & 6.1 & 24 & 4.8 \\
\hline 22 & high & high & 10 & 10 & 131 & 3802.1 & 12 & 2.8 & 6 & 0.7 \\
\hline 23 & high & high & 20 & 5 & 133 & 3616.2 & 18 & 4.5 & 10 & 2.6 \\
\hline 24 & high & high & 20 & 20 & 111 & 1808.0 & 5 & 3.7 & 0 & 0.0 \\
\hline
\end{tabular}

Each of the 24 sets is optimized assuming that no sampling inspection is in effect. Table 2 reports the optimal procurement quantity along with the corresponding expected profit. Moreover, assuming that there is the alternative of conducting a sampling inspection before the determination of $\mathrm{Q}$, we specify the optimal sample size, $\mathrm{n}^{*}$, using the same 24 parameter sets including the sample inspection cost, $c_{n}$, which is examined in two levels, low $\left(c_{n}=c_{d a}\right)$ and high $\left(c_{n}=2 c_{d a}\right)$. The resulting optimal values of $\mathrm{n}$ and the corresponding profit increase as compared to the no-sampling case are also shown in Table 2. It should be noted that when sampling inspection is allowed, the optimal procurement quantity value depends on the inspection outcome, $\mathrm{Q}_{\mathrm{n}}$. Thus, for a given sample size, $\mathrm{Q}^{*}$ is a function of $\mathrm{Q}_{\mathrm{n}}$, and it is evaluated through the maximization of (5).

Examination of Table 2 reveals that the introduction of sample inspection may increase the profitability of remanufacturing. This improvement is attributed to the fact that after inspection the additional information on returns' yield allows the more precise determination of the procurement quantity. For example, for set \#12 (high $\mathrm{c}_{\mathrm{n}}$ ) with $n^{*}=2$, if $Q_{n}=2$ the optimal procurement quantity is $Q^{*}=130$, if $Q_{n}=1$, then $Q^{*}$ $=129$ and if $\mathrm{Q}_{\mathrm{n}}=0$, then $\mathrm{Q}^{*}=0$. On the other hand, based solely on the prior to inspection knowledge, the optimal decision would be to procure $\mathrm{Q}^{*}=125$ units. This effect is more pronounced for sets \#4 (low $c_{n}$ ) and \#8 (for both low and high values of 
$\left.c_{n}\right)$. For these sets in the no-sampling case $Q^{*}=0$, while with sampling inspection there can be the opportunity to procure some quantity leading to positive expected profit (45.6 for set \#4 and 253.5 or 171.0, respectively for set \#8). However, in set \#4 (high $c_{n}$ ) the value of information from sampling vanishes because of the high value of $c_{n}$. Another consequence of inspection sampling is that it can prevent remanufacturing firms from procuring inferior-quality lots. For example, in case \#1 (low $c_{n}$ ) without sampling $\mathrm{Q}^{*}=205$, while when sampling is allowed, for $\mathrm{Q}_{\mathrm{n}}<8$ it is optimal not to procure at all $\left(\mathrm{Q}^{*}=0\right)$.

Based on the results, we conclude that high sampling cost decreases the value of $\mathrm{n}^{*}$, reducing sampling discriminatory power; thus, the value of sampling decreases. Sampling inspection is more advisable, in terms of percentage profit improvement, when the expected yield of returns is rather low. On the contrary, when yield variability is low, the benefits of sampling inspection decrease or even vanish. The impact of $\mathrm{c}_{\mathrm{da}}$ differs depending on the expected yield of returns. Specifically, for low or medium values of $E(q)$, the value of sampling inspection increases with $c_{d a}$, since performing an initial quality assessment of the returns decreases the amount of nonramanufacturable units disassembled and disposed of. On the other hand, when the returns' expected yield is high, the percentage profit improvement due to sampling inspection is perceivable only for low values of $c_{d a}$ (mainly as a result of lower $c_{n}$ values). Finally, based on the numerical examples studied we conclude that the value of $\mathrm{c}_{\mathrm{r}}$ does not influence notably the results.

\section{Summary and Future Research}

In the current paper we examined the advisability of establishing sampling inspection prior to the determination of the procurement quantity in a reverse supply chain. We formulated the expected profit functions for the cases with and without sampling inspection and evaluated the optimal decisions under different values of the problem's parameters.

The most important contribution of the current paper is that it proposes a new method for resolving, at a certain extent, the uncertainty regarding returns' quality which is inherent in reverse supply chains. Moreover, it enables the evaluation of the economic benefits of sampling inspection and the determination of the optimal values of the sample size and the procurement quantity with respect to the sampling outcome.

It has been shown that the establishment of sampling inspection can substantially improve the profitability of a reverse supply chain. The outcome of sampling inspection allows the remanufacturer to refine the procurement quantity decision and also to avoid procurement of lots characterized by low returns' yield. The optimization results provide insights on the factors that affect the value of sampling inspection before the procurement of returns. Specifically, through the numerical investigation presented we found that the advisability of establishing sampling inspections is increasing as the quality uncertainty and the disassembly cost increase and as the expected yield of returns and the sampling inspection cost decrease.

Interesting extensions of the proposed model include the investigation of the impact of different degrees of sampling inspection accuracy and of the advisability of 
sampling inspection when multiple returns quality states and recovery options exist. In the latter case the inspection outcome would define, apart from the procurement quantity, the appropriate recovery process, as well.

\section{References}

1. Guide Jr., V.D.R., Van Wassenhove, L.N.: Managing product returns for remanufacturing. Production and Operations Management 10(2), 142-155 (2001)

2. Debo, L.G., Savaskan, R.C., Van Wassenhove, L.N.: Coordination in closed-loop supply chains. In: Dekker, R., Fleischmann, M., Interfurth, K., Van Wassenhove, L.N. (eds.) Quantitative Models for Closed Loop Supply Chains, pp. 295-311. Springer, Berlin (2004)

3. Guide Jr., V.D.R., Souza, G.C., Van Wassenhove, L.N., Blackburn, J.D.: Time value of commercial product returns. Management Science 52(8), 1200-1214 (2006)

4. Geyer, R., Neeraj, K., Wan Vassenhove, L.N.: Reverse logistics in an electronic company: the NEC-CI case. In: Flapper, S.D.P., Van Nunen, J.A.E.E., Wan Vassenhove, L.N. (eds.) Managing Closed-Loop Supply Chains, pp. 33-39. Springer, Berlin (2005)

5. Driesch, H.M., Van Oyen, Flapper, S.D.P.: Recovery of car engines: the Mercedes-Benz case. In: Flapper, S.D.P., Van Nunen, J.A.E.E., Wan Vassenhove, L.N. (eds.) Managing Closed-Loop Supply Chains, pp. 157-166. Springer, Berlin (2005)

6. Nenes, G., Panagiotidou, S., Zikopoulos, C.: Procurement and sampling decisions under stochastic returns yield in reverse supply chains. Working Paper (2009)

7. Ferrer, G.: Yield information and supplier responsiveness in remanufacturing operations. European Journal of Operational Research 149, 540-556 (2003)

8. Ferrer, G., Ketzenberg, M.E.: Value of information in remanufacturing complex products. IIE Transactions 36, 265-277 (2004)

9. Aras, N., Boyaci, T., Verter, V.: The effect of categorizing returned products in remanufacturing. IIE Transactions 36, 319-331 (2004)

10. Galbreth, M.R., Blackburn, J.D.: Optimal acquisition and sorting policies for remanufacturing. Production and Operations Management 15(3), 384-393 (2006)

11. Ketzenberg, M.E., Van der Laan, E., Teunter, R.H.: Value of information in closed loop supply chains. Production and Operations Management 15(3), 393-406 (2006)

12. Souza, E.A., Ketzenberg, M.E., Guide Jr., V.D.R.: Capacitated remanufacturing with service level constraints. Production and Operations Management 11(2), 231-248 (2002)

13. Tagaras, G., Zikopoulos, C.: Optimal location and value of timely sorting of used items in a remanufacturing supply chain with multiple collection sites. International Journal of Production Economics 115, 424-432 (2008)

14. Yano, C.A., Lee, H.L.: Lot sizing with random yields: A review. Operations Research 43(2), 311-334 (1995)

15. Lee, H.L., Rosenblatt, M.J.: Optimal inspection and ordering policies for products with imperfect quality. IIE Transactions 17(3), 284-289 (1985)

16. Ben-Daya, M., Noman, S.M.: Integrated inventory and inspection policies for stochastic demand. European Journal of Operational Research 185, 159-169 (2008) 